\title{
Is Scalability Necessary for Economic Sustainability?
}

\author{
By Dr. Dennis F.X. Mathaisel
}

\begin{abstract}
The objective of this paper is to investigate the impact of scalability on the sustainability of any entity (ecological, environmental, human, or enterprise) anywhere. Scalability refers to the ability of the enterprise to grow without losing customers, diminishing quality, or changing the core value proposition of the organization. The question to be addressed is whether or not growth is necessary. The author has developed a framework for a sustainable entity that addresses five abilities for an entity to be sustainable: availability, dependability, capability, affordability and marketability. Scalability, as an addition to these five abilities, represents a unique challenge for some institutions, especially small ones. Scalability attempts to describe the sensitivity of the entity to changes in the scope of operations, specifically in the context of SMEs (Small and Medium sized Enterprises) because their challenges are unique. Larger organizations have the luxury of established practices, cultures, and recognition. Small businesses and new ventures face an environment where their brands and cultures may still be evolving, and the quality of their products and services could change with increases in scale. Consequently, this paper explores the theory that entities may not be scalable because, through growth, they might intrinsically change their core businesses and suffer losses.
\end{abstract}

Keywords: economic sustainability, scalability, SME sustainability, sustainability abilities, small business growth.

\section{Introduction}

A common principle of modern business thinking is that growth is good. It is usually thought that all businesses should seek to increase their revenues and gain market share. It is the purpose of this paper to show that some businesses, especially small ventures with unique value propositions, should not necessarily seek to grow or scale up. There are numerous examples of new ventures failing for various reasons, and many of these have to do with growth. While new ventures frequently offer creative, innovative solutions to market needs, there are also aspects of these businesses that will be crucial to maintain while scaling up. Consequently, some new ventures may succeed based on aspects of the organization that are not feasible to scale, and thus a more conservative growth strategy should be undertaken.

Scalability refers to the ability of a business to grow without losing customers, diminishing quality, or changing the core value proposition of the organization. In other words, it is developing products or services that people want and figuring out how to produce many of them for lower costs while selling more of them (Dudnik 2010). Scalability attempts to describe the sensitivity of businesses to changes in their scopes of operations, specifically in the context of small businesses because their challenges are unique. Larger organizations have the luxury of established business practices, corporate cultures, and brand recognition. Small businesses face an environment where their brands are still being formed, their cultures may still be evolving, and the quality of their offerings could change drastically with increases in scale or volume (Rogers 2014).

This paper explores the theory that some entities may not be scalable. To explore this hypothesis, the author developed a framework for sustainability that addresses five abilities - availability, 
dependability, capability, affordability and marketability - with scalability as an addition to these five.

\section{A Framework for a Sustainable Entity}

A framework for sustainability (Figure 1) has been developed (Mathaisel et al 2009) that consists of five abilities: Availability, Dependability, Capability, Affordability, and Marketability. Availability refers to the resources needed to run the entity, such as people, technology, facilities, and parts. It represents the ability of a venture to survive based on a sustained source of inputs. As an example, supply chain availability consists of three principles: integration, collaboration, and trust, which are all required for sustainability. Dependability is described as the science of performing without failure, degradation, or placing high demands on the support infrastructure. It includes reliability, maintainability, supportability, and testability. Dependability also represents the ability of a venture to survive as a result of its continued delivery of services or products. Capability is the ability to identify and fulfill performance metrics, performance measurement, and performance management for assessing the effectiveness of the service or product. It represents a venture's success against a measurable framework, which inherently involves defining and adhering to critical measures of success. Affordability concentrates on the methods and models for analyzing and improving the life cycle cost (LCC) of an entity's products. Affordability identifies just what costs will be involved throughout the life of the product. This ability is crucial for small ventures as they attempt to estimate capital needs on a long-term horizon with growth challenges. Marketability describes the aspect of a business that relates to identifying the target area (market) for a sustainable product, service, or entity, and enhancing the methods and models to serve that market. It is the ability of the enterprise to motivate change and to market itself, which is crucial to its viability, and is much more than just selling.

\section{Literature Review}

Blumberg and Hindi (2013) recently published Startup CEO: A Field Guide to Scaling up Your Business that describes five main facets to scaling a business. Focusing on storytelling, building human capital, execution, the board of directors, and personal management, Blumberg and Hindi imply that all businesses are scalable, given careful growth management. The first two considerations, storytelling and building human capital, address early-stage components of scaling a business. Explaining the importance of developing a compelling and well-meted story, Blumberg and Hindi suggest extensive testing and explanation of why a business was started. Building human capital addresses the needs of a growing business to develop a corporate culture, and structure payment, promotion, and firing of personnel. The Execution component of Blumberg and Hindi's guide is of interest because it addresses the specifics of raising capital for growth, structuring a "Company Operating System," and managing the company through troubled waters. The last two tenants of the book focus on building and managing a "Personal Operating System," which they use to describe a framework for personal management. 


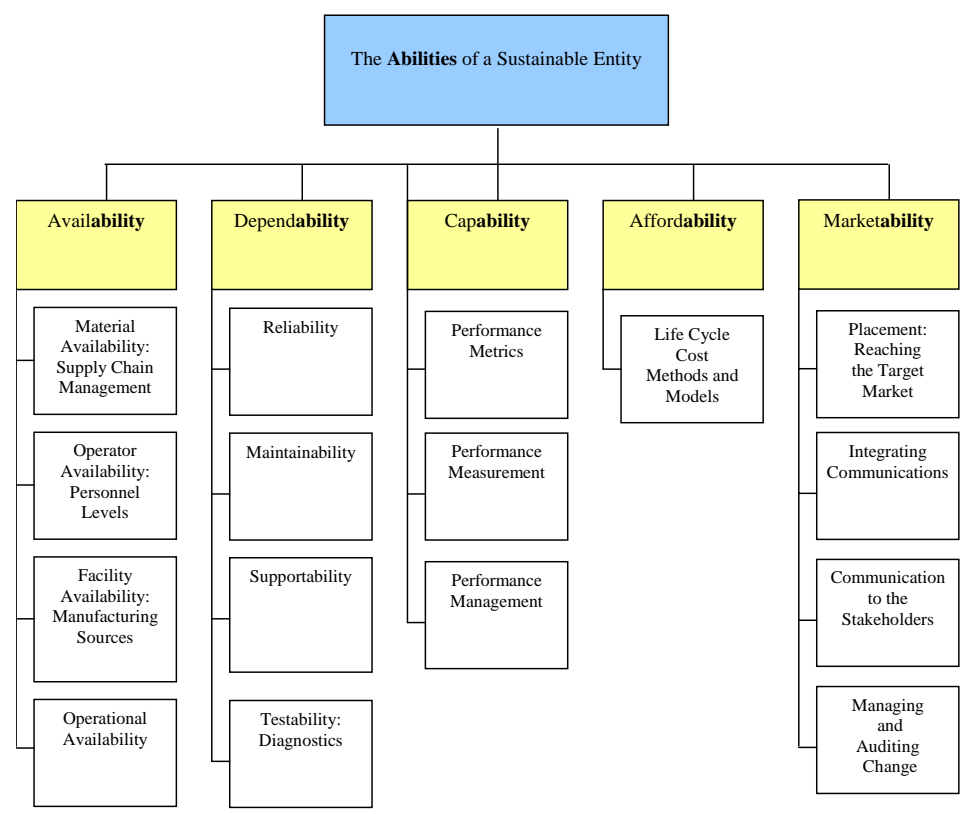

Figure 1: The Five Abilities of a Sustainable Entity

Gartner et al (1998) offer a critical analysis of the predictions of Inc. Magazine's list of new ventures versus those of a Venture Screening Questionnaire. The goal was to analyze the effectiveness of the questionnaire compared to Inc. Magazine's determinants of new venture performance. The criterion for determining if a new venture had survived was if the business was still in operation over a four year timeframe. New ventures that survived were more likely to have entrepreneurs who: gained knowledge and ability during the founding process; devoted greater efforts to dealing with suppliers; analyzed potential new entrants; and devoted less time to determining the identity of the business. Survival was also more likely for businesses that had: fundable resource requirements; focused on products or services that were designed or produced to order; and were in high growth industries. Of the 27 businesses profiled at the beginning of the comparison, 17 were still in operation four years later.

The Small Business \& Entrepreneurship Council (2011) published the "Small Business Survival Index 2011", which describes the overall climate for small businesses in every state in the United States. Focusing on the impact of public policy, such as taxes, regulatory costs, government spending, and energy costs, the report covers 44 individual factors. One aspect of the research conducted by the Small Business \& Entrepreneurship Council is the correlation that they found between sustainability costs and small business survival. A March 2012 survey that they cite in TechnoMetrica states that 41 percent of small business owners said high sustainability costs were affecting their plans to hire.

Sutton and Rao (2014) examined over 400 companies. Their premise was that, although situations vary greatly, there are common lessons learned. One is that many entrepreneurs increase their scale too much, too soon. They also found that it is important to take the time to do things right, and forming a culture is important for success. Emphasizing the need for adaptive practices, they advocate flexible practices and roles for the sake of fostering an empowering culture. To achieve this goal it is important to use "hierarchy to defeat bureaucracy." They claim that more knowledge 
at the start of a new venture can actually be a hindrance because of biases and the "success syndrome." However, an inexperienced entrepreneur may in fact be more receptive to iterative learning and open-mindedness.

Kumar (2010) found that growing or scaling a business can be approached from two main perspectives regarding the timing of expansion. The question is a paradox: whether to grow in advance of demand with the hopes of capturing maximum market share and minimizing foregone revenue; or, more conservatively, does the business grow when demand already exists? Increasing the scale of a business in advance of real demand relies on careful forecasting and represents a greater risk. However, waiting to grow could result in the business losing customers to competitors and potentially missing an entire swing in the market. While many factors go into the decision to grow a business, forecasts are ultimately only accurate to a certain extent, and as new ventures are formed they must decide how they will approach such an important part of the life of their business. Consequently, the environment of forecasting and planning has changed because forecast-based planning methods and/or budget-oriented planning are alone not sufficient for any business to be sustainable.

Walsh (2015) refers to MetLife insurance suing a panel of regulators to fight its designation as "systemically important," making it the first financial company to go to court over the issue of scale since the government started singling out too-big-to-fail institutions in an effort to stem future financial crises. MetLife did not like the negative connotation of being singled out for stricter oversight, while other similar businesses were not. Can scalability be a liability for an entity like MetLife?

\section{Scalability}

This paper examines scalability as an addition to the five abilities framework for sustainability (see Figure 2). Is scalability a necessary requirement for a sustainable entity? Scalability is "the ability of a system to accommodate increasing numbers of users without unacceptable levels of performance degradation" (Webster 2014). Kumar (2010) notes that scalability of an entity begins by addressing four concepts: customer value proposition (CVP), business attribute analysis, change capacity, and market analysis. Understanding CVP is an important determinant of scalability, because it reveals just how the entity is adding value while driving return business. Changing CVP can reduce the customer base and diminish branding. Business attribute analysis reflects the need for the entity to gain an awareness of just what differentiates it from the competition. Change capacity can be used to evaluate scalability. Similar to the capability and availability abilities of Figure 1, change capacity specifically looks at the tradeoffs of increasing capacity versus scalability. Many entities benefit by being able to offer high levels of customer support, flexibility, customization, and response, while change capacity seeks to address just how much scaling can be accommodated before any of these core attributes begin to diminish. Kumar refers to capacity of this nature in stating that high performance entities rely on high-performance people. 


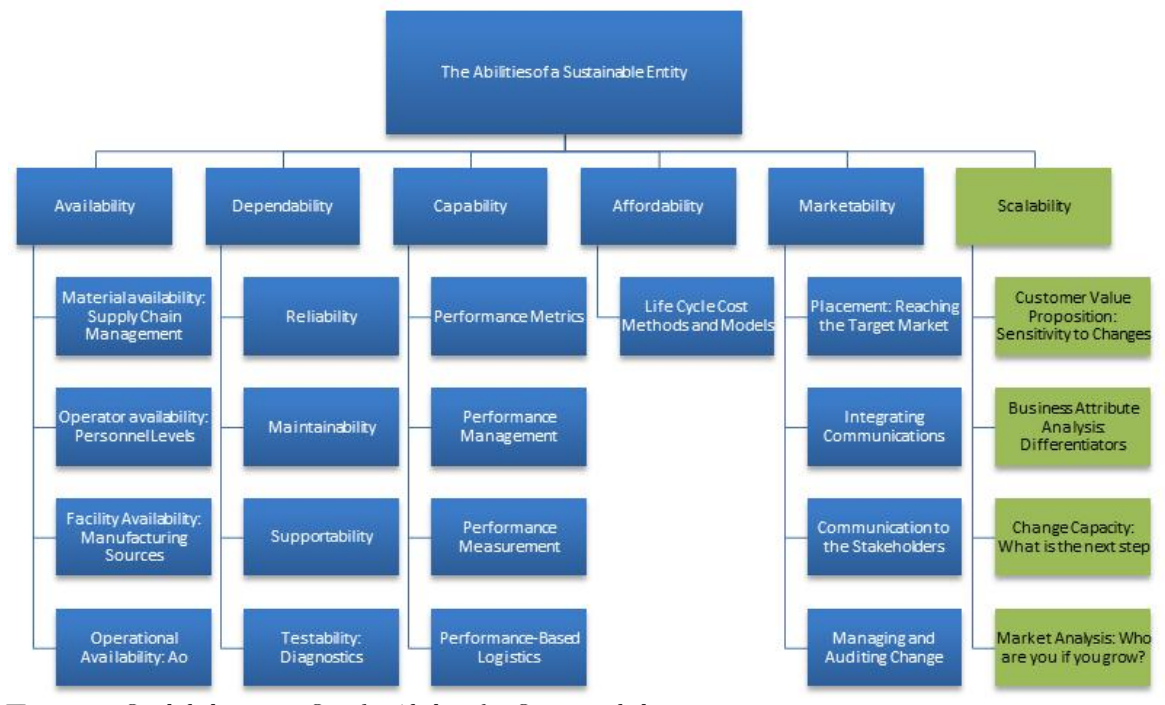

Figure 2: Scalability as a Sixth Ability for Sustainability

\section{Case Studies in Scalability}

Sustainable Minds (2015) offers cloud-based software that enables product designers to measure the environmental impact of their products. A derivative of life-cycle analysis software, the Sustainable Minds platform is designed to allow non-engineers to begin implementing ecodesign concepts. As an organization, Sustainable Minds also offers services that accompany the design of greener products. With five employees, Sustainable Minds is an example of an organization that struggles with scalability. The CEO, Terry Swack, leads the company and oversees most business development actions, operations, and strategy. Michael Potts, the only other full-time employee, leads the sales force of occasional interns. Terry's life-cycle analysis expert, software designer, and Chief Technology Officer are on retainers and located across the United States. To many newcomers, Sustainable Minds appears to be an organization on the verge of major growth. It is always entering new markets and landing major accounts. However, it falls short of its growth goals. The question is if lack of scalability is an issue for Sustainable Minds. For example:

a. Value in the organization lies with the CEO's knowledge of Eco design concepts and her ability to sell these designs.

b. Terry's approach to business development and acquiring capital has been the lifeblood of the company. Scaling the business will require even more of her time.

c. Sustainable Minds struggles to address the different needs of new markets, like commercial markets for practical uses.

d. Sustainable Minds' past success has been its stature as a small business, and as it grows it may lose this component and not attract new potential customers.

Clean Bottle (2015) offers a line of water bottles that come apart at both the top and bottom for thorough cleaning and ease of maintenance. Clean Bottle was born of the increasingly popular "crowd funding." Crowd funding is a model of bringing new products to market with individuals pledging money, but their funds are only used if enough pledges are made to reach a specific threshold. Clean Bottle set the goal for their crowd funding campaign at $\$ 20,000$, and nearly doubled it before their deadline. Clean Bottle is a successful example of scalability because it offers a product that requires little inputs beyond basic manufacturing. In their quest for meeting 
capacity, Clean Bottle has four different factories, two patents, and three years of careful growth. From the perspective of scalability, producing a durable product is one of the easiest business models to use. With an established design and Bill of Materials, Clean Bottle only needed to find the correct manufacturer that met its standards. Obstacles occur for a growing business when manufacturers operate only at large scale production levels or are inflexible regarding new partners. This is probably why Clean Bottle over time used four different manufacturers. Blumberg's (2013) "Scaling up a Business Framework" applies to Clean Bottle:

a. Storytelling: Clean Bottle has a compelling story of discovering a moldy water bottle and wishing that it could come apart for cleaning. They brought the product to life in their crowd funding campaign through story telling - friends wanting to help the world have cleaner water bottles.

b. Execution: The Clean Bottle team created the design, tested it, and built a prototype. However, they needed capital to bring it to scale. Crowd Funding was their chosen venue - the crowd helped design the campaign and the web-site.

c. Board of Directors: Dave Mayer is a long-time Ironman Triathlon competitor and amateur cyclist, so sustainability runs in his blood. Formerly part of the investment company of Goldman Sachs and also a product manager for Cisco, Mayer was not an inexperienced entrepreneur.

Greyston Bakery (2015) was founded in 1982 in New York. An organization with a social mission, Greyston Bakery trains and employs people with diverse backgrounds, including convicts and the homeless, and even helps them work towards attaining GEDs (General Educational Development tests) (Adams 2013). Greyston Bakery is the main supplier of brownies to Ben and Jerry's, and thus faces a unique scalability challenge. Intrinsic to their mission is using by-hand baking techniques as a means to facilitate high levels of employment and ensure quality control. However, as demand continues to increase and they struggle to meet orders, Greyston must make a critical decision about its scale of operations. If the organization decides to change capacity, they will be forced to cut back on staffing levels, which contradicts their mission of employmentfirst.

The Etsy Store, Three Bird Nest (2015), is an online marketplace for handmade goods. Its founder, Alicia Shaffer, has been mass producing some of her goods and procuring wares from China. Because of the great increase in sales volume, the authenticity of her handmade goods is being questioned. One quote from a longtime Etsy seller is “handmade businesses aren't infinitely scalable, just by the definition of the term" Three Bird Nest (2015).

\section{Scalability across Different Entities}

The scalability of businesses is affected by the industries in which they operate. Some business models are easier to scale, while others can be much more challenging. As found by Sutton and Rao (2014), culture and structure are often more important than anything else. Businesses that produce a tangible product, such as the Clean Bottle case and the Greyston Bakery case, or most types of consumer purchased goods, are inherently simpler to scale. Small ventures of this type have many opportunities to achieve economies of scale, cost savings, and increased efficiencies. However, scaling up to achieve greater efficiencies and production levels exposes the entity to problems, such as decreased quality, lessened control over processes, and increased complexity. Another important aspect of scaling up for small businesses is customer value perception (CVP), where perceptions of organizations can change when they grow. The case of the Etsy Store Three Bird Nest illustrates CVP.

A classic question for manufacturers of high quality goods is how do they maintain standards while meeting increased demands? Relyea (2013) addresses these concerns: "All processes are the same in that they consist of equipment, raw material, people, and methods. Yet, each process is 
unique in terms of the variation that is inherent to a specific process. Understanding the nature of the variation that is unique to a specific process is the key to applying classic problem-solving tools that have been proven to be effective regardless of what is being manufactured."

Entities that offer services, particularly small businesses, are more complicated to scale. Both product and service entities require intelligent leaders, but it is this aspect of the organization that can make a service-oriented new venture challenging to scale. Bringing unique intelligence, experience, and passion to the organization, a small venture offering services must maintain these factors as they scale up. Services offered by small ventures are known for being customizable, personal, flexible, and of high quality. The case of Sustainable Minds serves as an excellent example of this challenge. With high levels of participation from a founding entrepreneur, scaling up requires careful attention to just what behaviors are leading to the success of the organization and how they can be replicated. Building a brand that represents such qualities can afford an organization some slack as it grows. However, a failure to deliver on such expectations will not provide sustainability. In Paul Niven's book (2006), Margaret Blair of the Brookings Institute says: "If you just look at the physical assets of the companies, the things that you can measure with ordinary accounting techniques, these things now account for less than one-fourth of the value of the corporate sector. Another way of putting this is that something like $75 \%$ of the sources of value inside corporations is not measured or reported on their books."

\section{Conclusions}

As a sixth addition to the Abilities Framework, Scalability describes an aspect of many small businesses that often involves intangible influences. Frequently focused on growth and increased revenue, small businesses offer an intrinsic value that is easily overlooked. Providing flexibility, customizability, personality, and quality, small businesses need to consider that some aspects of their value proposition might be irreplaceable in growth. The very nature of small businesses and their approaches to value can represent traits desired by consumers. Therefore, sustainable and consistent value in some cases will be achieved only through maintaining current business operations and foregoing growth opportunities.

Views of business models continue to change, and with a growing concern about small business survival in the face of big business economies of scale, the importance of recognizing just where a business belongs in terms of its scale is more essential. Adding Scalability to the Abilities Framework will help to measure an aspect of new venture sustainability that attempts to explain a dichotomy between small ventures and their larger established counterparts. A conclusion can be reached that the spirit and energy that enabled small entrepreneurs to start businesses are so difficult to replicate that some businesses might not be scalable without changing the aspects that define them.

\section{References}

Adams, Susan (2013). "12 Companies Considered 'Best for the World'." Forbes. http://www.forbes.com/sites/susanadams/2013/04/17/12-companies-considered-best-for-the-world/. Retrieved from the web on 16 March 2015.

Blumberg, Matt and Hindi, Hanny (2013). Startup CEO: A Field Guide To Scaling Up Your Business, Wiley. ISBN 9781118683279. September.

Clean Bottle (2015). http:/ /www.cleanbottle.com. Retrieved from the web on 13 March 2015.

Dudnik, Nina (2010). "Social Entrepreneurs' Tricky Issues of Sustainability and Scale.” Harvard Business Review. 
Gartner, William B., Starr, Jennifer A., Bhat, Subodh (1998). "Predicting new venture survival: An analysis of 'anatomy of a start-up' cases from Inc. magazine.” Journal of Business Venturing. Elsevier. 14. pp. 215-232. March.

Greyston Bakery (2015). http://greyston.com/about-greyston/news-press/. Retrieved from the web on 17 March 2015.

Kumar, Dhirendra (2010). Enterprise Growth Strategy: Vision Planning and Execution. Ashgate Publishing Group.

Mathaisel, Dennis F.X, Manary, Joel and Comm, Clare L (2009). Enterprise Sustainability. CRC Press. Taylor and Francis.

Mathaisel, Dennis F.X, Comm, Clare L (2011). “A Strategy for Enterprise Sustainability.” Business Review Cambridge. Vol. 17, No. 1. Summer.

Merriam-Webster (2005). The Merriam-Webster Dictionary. ISBN-13: 978-0877796367 ISBN10: 087779636X. Merriam Webster. New edition. January.

Niven, Paul (2006). Balanced Scorecard Step-by-Step: Maximizing Performance and Maintaining Results. John Wiley \& Sons.

Parnell, John A. (2008). "Sustainable Strategic Management Construct, Parameters, Research Direction," International Journal of Sustainable Strategic Management. Vol. 1, No. 1.

Relyea, Douglass B. (2013). Popular Manufacturing Myths: Eliminating Widely Held Beliefs that Reduce Competitiveness. Productivity Press.

Reinhardt, F. (2000). "Sustainability and the Firm.” Interfaces. Vol. 30, No. 3. pp. 26-41.

Rogers, Kate (2014). "Scaling Up Lessons from Google and Facebook." http://smallbusiness.foxbusiness.com/starting-a-business/2014/02/06/scaling-up-lessons-fromgoogle-and-facebook/.

Rothwell, William J. (2010). Effective Succession Planning: Ensuring Leadership Continuity and Building. Talent from Within. AMACOM. Fourth Edition.

Small Business \& Entrepreneurship Council (2011). "Small Business Survival Index 2011." Ranking the Policy Environment for Entrepreneurship Across the Nation. November.

Sustainable Minds LLC. (2015). http://www.sustainableminds.com. Retrieved from the web on 10 March 2015.

Sutton, Robert., Rao, Huggy (2014). Scaling Up Excellence: Getting to More without Settling for Less. Crown Publishing Group.

Tabuchi, Hiroko (2015). "Armies of Artisans: A Market and Its Vendors Run Into Problems of Scale and Credibility.” New York Times. March 16. B1-B3.

Three Bird Nest (2015). https://www.etsy.com/shop/ThreeBirdNest. Retrieved from the web on 15 March 2015.

Walsh, Mary Williams (2015). “MetLife Sues Over Being Named Too Big to Fail.” New York Times. January 14. B6-B7. 